\title{
A service evaluation exploring the effectiveness of a locally commissioned tier 3 weight management programme offering face-to-face, telephone and digital dietetic support
}

Rosemary Huntriss ${ }^{a^{*}}$, Matthew Haines ${ }^{b}$, Lucy Jones ${ }^{a}$ and Danielle Mulligan ${ }^{\mathrm{C}}$

a Nutrition and Dietetics, Oviva UK Ltd, London, United Kingdom

${ }^{b}$ Department of Allied Health Professions, Sport and Exercise, University of Huddersfield, Huddersfield, United Kingdom

${ }^{c}$ Nutrition and Dietetics, Manchester University NHS Foundation Trust

Keywords

- Weight management

- Digital health

- Telehealth

- Dietetics

- Nutrition

* Corresponding author:

Rosemary Huntriss

Address:

Email address: 


\section{INTRODUCTION}

34 The challenge of obesity management is a major public health concern. Most adults in England (64\%) are affected by overweight or obesity, with the prevalence of obesity continuing to rise. ${ }^{1}$ The personal and economic burdens associated with this are well known. Adults who are affected by overweight or obesity are at a much higher risk of developing conditions including type 2 diabetes, heart disease, stroke and some cancers. ${ }^{2}$ The associated annual National Health Service (NHS) costs attributed to overweight and obesity are projected to reach £9.7 billion by 2050 , with wider costs to United Kingdom (UK) society predicted to reach $£ 49.9$ billion. ${ }^{3}$

In the UK, there are four tiers of weight management service to support people who are affected by overweight or obesity 1) universal interventions which typically include identification and reinforcement of healthy eating and physical activity messages, offered population-wide; 2) lifestyle interventions and pharmacotherapy often as part of a multicomponent weight management service; 3) specialist services inclusive of a multi-disciplinary team (MDT) and specialist assessment; and 4) pre-operative assessment with the possibility of bariatric surgery supported by a multidisciplinary team. ${ }^{4}$ The National Institute for Health and Care Excellence (NICE) guidelines state that referral to a tier 3 service should be considered if the underlying causes of obesity need to be assessed; if the person has needs that cannot be managed adequately in tier 2 weight management services; if conventional treatment has been unsuccessful; if drug treatment is being considered for a person with a Body Mass Index (BMI) greater than $50 \mathrm{~kg} / \mathrm{m}^{2}$; or if bariatric surgery is being considered. ${ }^{5}$

54 Traditional tier 3 services have typically been delivered as face-to-face interventions ${ }^{6-8}$ and evidence suggests that programmes that provide contact with a dietitian are associated with greater weight loss. ${ }^{9}$ However, face-to-face care can be inconvenient for patients which might

57 increase attrition ${ }^{10}$ and can also be time-, cost-, and resource-intensive. Furthermore, evidence suggests that frequent support can enable more effective behaviour change ${ }^{11}$, which 
can be challenging to achieve with face-to-face care. To parry this, focus has shifted to the advancement of interactive digital technologies, with the potential for lower-cost scalability, as a means to enable health systems to better manage a growing and ageing population with an increasing presence of obesity-related co-morbidities. ${ }^{12-13}$ For example, several recent systematic reviews and meta-analyses have demonstrated that mobile phone interventions can result in modest weight loss and might therefore be a useful tool for promoting weight loss among adults who are affected by overweight or obesity. ${ }^{14-16}$

Furthermore, we have seen a significant increase in demand of telehealth technologies to support healthcare in recent times due to the health and safety risks posed to patients and healthcare professionals by the COVID-19 outbreak. The COVID-19 crisis has led to extraordinary transformations of service delivery using telehealth technologies, leading to a conception of the likelihood of their continued heightened presence post COVID-19. ${ }^{17}$

Thus, digital healthcare has the potential to improve safety, efficiency, accessibility and personalisation of healthcare interventions, ${ }^{18}$ but it is imperative to consider the context within which these interventions are delivered. Studies examining efficacy under controlled circumstances are frequently impractical and uneconomical in real-world interventions and do not necessarily establish their effectiveness in free-living conditions. ${ }^{19}$ These concerns are potentially decisive because effectiveness is what matters to patients and commissioners. Yet to date there has been no evaluation of a tier 3 weight management service offering traditional methods and digital service provision. Therefore, the purpose of this study was to evaluate the effectiveness of an aspect of a locally commissioned tier 3 weight management programme offering face-to-face, telephone, and digital (smartphone application) support and to compare the effectiveness of each intervention in everyday practice.

\section{MATERIALS AND METHODS}

83 The aim of this observational study was a retrospective examination of weight change in 84 patients referred to an NHS weight management programme. The study was a service 
evaluation and was carried out after institutional ethical approval. No data were collected outside of routine clinical care and all databases were anonymised.

\section{$87 \quad$ Participants}

88

Outcome data were collected for patients attending the Way to Wellness programme provided by Oviva UK Ltd. as part of a tier 3 weight management programme based in Wakefield, UK, which was jointly commissioned by the NHS and local authority. Due to local commissioning specifications, slight variations to the British Obesity \& Metabolic Surgery Society (BOMSS) commissioning criteria were observed; and other elements of the tier 3 service (NHS Consultant provision and physical activity services) were delivered by other providers. Eligible patients were over the age of 18 -years with a BMI $\geq 45 \mathrm{~kg} / \mathrm{m}^{2}$ or $\geq 40 \mathrm{~kg} / \mathrm{m}^{2}$ with a complex comorbidity. In exceptional circumstances, patients were considered eligible if they did not meet the BMI criteria, but it was agreed by the local commissioner and programme provider that weight management support from other tiers would be inadequate for their needs. Exclusion criteria for this service included patients with an active eating disorder, unstable medical condition, unstable psychiatric disorder, women who were pregnant or breastfeeding, and patients who were not ready to change, defined by those who did not sign a pledge to declare their commitment to the programme. Patients included in the evaluation started care from $1^{\text {st }}$ January 2018 and were discharged from the core programme before $31^{\text {st }}$ December 2018.

\section{Procedures and outcomes}

Patients first attended a consultation and medical review with an NHS Consultant Physician. During this consultation, obesity-related comorbidities were screened for and investigated where appropriate.

\section{$\underline{\text { Core programme }}$}


109 Patients were then offered an initial 45-minute face-to-face consultation and a final 30-minute

110 face-to-face session with a Specialist Weight Management Dietitian. Between these sessions,

111 patients were offered one of three treatment interventions: 1) four 30-minute face-to-face

112 appointments; 2) two hours of online coaching using the Oviva smartphone application (app)

113 approved by NHS Digital; or 3) four 30-minute telephone appointments. All care within the

114 core programme was delivered by the same dietitian and all patients received the same

115 amount of clinician time within their care. The choice of treatment intervention was that of the

116 clinician and patient according to patient preference and professional standards. The aim was

117 to attend the final session of each intervention within 12-16 weeks of commencement.

118 Flexibility was permitted to cater for changes to appointments and dietitian availability which

119 resulted in the mean duration of the interventions being $19 \pm 5$ weeks, $15 \pm 4$ weeks, and 17

$120 \pm 2$ weeks, for the Face-to-face, App and Phone interventions, respectively.

121 At the initial dietetic assessment, all patients undertook a full assessment and were able to 122 select from a variety of dietary interventions, with support from their dietitian to determine the 123 most appropriate approach. These included diet and lifestyle goal setting $(65.5 \%$ of total 124 participants), calorie counting (25.4\%), a low-carbohydrate diet $(2.4 \%)$, a partial meal 125 replacement plan (5.9\%) or intermittent fasting (i.e. 5:2 diet, 1.8\%). The proportion of patients 126 using each diet was similar between groups. Very low-calorie diets and total diet replacements were not supported in this programme.

For patients undertaking the Face-to-face and Phone interventions, follow-up appointments involved the patient and dietitian reviewing existing goals and progress and setting or reestablishing SMART goals at the end of every session.

131 Patients who were app coached were also guided by SMART goals. Patients that chose to be supported via online coaching were asked to track their food intake by uploading pictures of meals or snacks, or by sharing their daily intake through text messaging on the secure Oviva app. SMART goals were re-established when necessary. Patients were able to track their 
physical activity levels, and were encouraged to share their thoughts and feelings with their dietitian via the app throughout the intervention. Patients with diabetes were also able to track their blood glucose levels. The dietitian reviewed progress and offered guidance allowing 15 minutes of coaching each week, the time typically split between Monday and Friday for eight weeks, providing a total of two hours of app coaching which was equivalent to the time offered for appointments in the Face-to-face and Phone interventions.

141 All patients had the opportunity to access up to six psychology support sessions from a Clinical

142 Psychologist or Psychological Wellbeing Practitioner (45-minute initial consultation and 30minute follow-up consultations) which were delivered face-to-face or via telephone with the aim of focussing on the psychological factors that contribute to weight gain to support the client to meet their weight loss goals. The focus of these sessions was food, eating and exercise but frequently involved discussion of broader psychological factors when they were contributory to unsuitable eating behaviours. Patients also had the opportunity for referral to an exerciseon-referral scheme. Monthly multi-disciplinary team meetings were held in person to discuss relevant patient cases and included the tier 3 dietitian and clinical psychologist, Consultant Physician, in addition to tier 4 dietitians and clinical psychologist.

The primary programme outcome was change in body mass, and all patients were encouraged to aim for a $5 \%$ reduction in baseline values with completion defined as attendance of more than $50 \%$ of dietetic sessions, which was the locally agreed key performance indicator (KPI)

154 for the commissioned service. These values were recorded by the dietitian at baseline and at the end of the core programme using calibrated scales (Seca Ltd., UK). For those that did not attend the final face-to-face appointment, self-reported values were used if pictorial evidence of the scales was uploaded or the value was aligned with the patients' reported food intake, current progress, and weight trajectory. If a patient did not complete the core programme, baseline values were carried forward for analyses. Other outcomes of interest included change in BMI, intervention adherence (considered as the proportion of patients that completed $50 \%$ or attended $100 \%$ of dietetic sessions), the number of psychology support sessions undertaken, 
and a Family and Friends Test which provided a score out of ten to describe the patients' satisfaction with the service.

The programme was guided by NICE public health $(\mathrm{PH} 53)^{19}$ and obesity guidelines (CG189). ${ }^{5}$

Patients who completed and attended the final appointment of their core programme were offered the opportunity of a 12-week follow-up appointment. This appointment was booked at the final appointment within the core programme. The follow-up appointment was a prerequisite for those patients who wanted to be referred to tier 4 services for assessment for bariatric surgery, acting as a gateway to bariatric surgery, or optional for those wanting additional support. The follow-up appointment consisted of a 30-minute review by a nutritionist arranged 12 weeks after completion of the core programme. Body mass was also recorded at this time.

\section{Statistical analyses}

175 Data were extracted, verified, and anonymised from patient records and discharge letters by

176 the Programme Lead Dietitian. Analyses were completed on an intention-to-treat (ITT) basis,

177 with data imputed to account for people lost to follow-up for those who did not complete the 178 core programme using baseline observation carried forward (BOCF). For those who attended 179 the 12-week follow-up appointment, completer data only were analysed at this point due to it 180 being an optional review. Statistical analyses were carried out using IBM SPSS Statistics version 26 (IBM, Armonk, USA) with alpha set at $P \leq .05$. Before analyses, data were explored to check assumptions of statistical tests. Normal distribution of data and identification of outliers and extreme scores were assessed via visual inspection of histograms and box plots

184 and using the Kolmogorov-Smirnov and Shapiro-Wilk tests. The effects of different treatment interventions on outcomes were then assessed by fitting linear models. Two-way (group-bytime) repeated measures analysis of variance (RMANOVA) was used to compare differences 
187 in body mass and BMI at baseline and post-intervention for the core programme and 12-week

188 follow-up separately. The sphericity assumption was checked using Mauchly's test with

189 corrections (Greenhouse-Geisser when $\varepsilon<0.75$ and Hyunh-Feldt when $\varepsilon \geq 0.75$ ) applied to 190 the degrees of freedom when this was violated. Significant main effects were considered using 191 post-hoc Bonferroni-corrected pairwise comparisons to control for familywise error rate. Effect 192 sizes were quantified using the partial eta squared $\left(\eta^{2}\right)$ statistic to examine the magnitude of 193 differences between the three interventions, with values of $0.1,0.3$, and $>0.5$ considered to 194 be small, medium, and large effects, respectively. Prior to carrying out the RMANOVA's, 195 possible covariates (age) and factors (sex) - that were not part of the main service evaluation 196 but could influence the dependent variable - were included in a preliminary ANOVA analysis 197 to check for independence of the predictor variable and were found to be non-significant. The 198 Family and Friends Test (FFT) scores were compared using one-way independent ANOVA. 199 For binary outcomes (e.g. achieving 5\% weight loss or not), the effects of factors were tested 200 by Pearson Chi-squared tests. The relationship between weight losses and measures of 201 intervention adherence were examined by Pearson correlation coefficient or Spearman rank 202 order.

\section{$203 \quad 3$ RESULTS}

\section{Descriptive data}

205 One hundred and sixty-nine patients $(79.3 \%$ female $)$ volunteered to commence the 206 programme. For $14(8.3 \%)$ cases no measure was available upon completion of the core

207 programme, so baseline values were carried forward for analyses (see Figure 1). Most 208 patients self-selected the App intervention (64.5\%) over the Face-to-face (28.4\%) and Phone

$209(7.1 \%)$ interventions. For the whole cohort, the mean ( \pm SD) age was $46.6 \pm 13.8$ years, and 210 baseline body mass was $132.9 \pm 20.9 \mathrm{~kg}$. Mean baseline BMI was $48.3 \pm 6.2 \mathrm{~kg} / \mathrm{m}^{2}$ with $96.4 \%$ 211 of the participating population having a baseline BMI of $40 \mathrm{~kg} / \mathrm{m}^{2}$ or above (range $37.1 \mathrm{~kg} / \mathrm{m}^{2}$ 212 to $\left.66.2 \mathrm{~kg} / \mathrm{m}^{2}\right)$. Characteristics for patients in each treatment intervention are detailed in Table 
213 1. At baseline, $33 \%$ of patients were diagnosed with type 2 diabetes (26.6\% for the App group,

214 compared to $45.8 \%$ and $41.7 \%$ for the Face-to-face and Phone groups, respectively).

215 Furthermore, a small proportion of patients were prescribed medications for weight loss or

216 blood glucose control including orlistat (5.3\%), glucagon-like peptide-1 analogues (6.5\%) and

217 sodium-glucose co-transporter-2 inhibitors (4.1\%), but the proportion of patients using these

218 did not differ between groups suggesting no associated impact on outcomes. Patients lost to

219 follow-up were $4.2 \%(2 / 48), 6.4 \%$ (7/109) and 41.7\% (5/12) for the Face-to-face, App and

220 Phone interventions, respectively. Seventy participants (41.4\%) attended the optional 12 -

221 week follow-up appointment. This included 21 patients for the Face-to-face intervention, 46

222 for the App intervention, and three for the Phone intervention. Due to insufficient data, it was

223 decided to exclude the Phone intervention from the analyses for the 12-week follow-up.

224 Table 1 Patient characteristics and outcome measures by intervention at baseline and at completion of 225 the core programme. Data are presented as intention-to-treat using BOCF.

\begin{tabular}{|c|c|c|c|c|c|c|}
\hline & $\begin{array}{r}\text { Face } \\
(n \\
\text { (female }=\end{array}$ & $\begin{array}{l}\text { ace } \\
\text { le = 11) }\end{array}$ & (female $=$ & 9) & $\begin{array}{r}\mathbf{P} \\
(n \\
\text { (female }\end{array}$ & ale = 3) \\
\hline & Mean & SD & Mean & SD & Mean & SD \\
\hline Stature (m) & 1.65 & 0.09 & 1.66 & 0.09 & 1.65 & 0.10 \\
\hline Age (years) & 52.7 & 14.1 & $43.3^{*}$ & 12.4 & 51.9 & 15.6 \\
\hline Baseline mass $(\mathrm{kg})$ & 128.8 & 18.5 & 135.9 & 21.9 & 123.2 & 16.2 \\
\hline End mass (kg) & $123.5^{\star *}$ & 18.4 & $129.8^{* *}$ & 21.5 & $119.2^{* *}$ & 18.2 \\
\hline $\begin{array}{l}\text { Change in mass } \\
(\mathbf{k g})\end{array}$ & -5.3 & 5.5 & -6.1 & 4.9 & -4 & 5.3 \\
\hline
\end{tabular}




\begin{tabular}{|c|c|c|c|c|c|c|}
\hline $\begin{array}{l}\text { Percent change in } \\
\text { mass }\end{array}$ & $4.1 \%$ & - & $4.5 \%$ & - & $3.4 \%$ & - \\
\hline $\begin{array}{l}\text { Baseline BMI } \\
\left(\mathrm{kg} / \mathrm{m}^{2}\right)\end{array}$ & 47.4 & 5.7 & 48.9 & 6.4 & 45.3 & 6.2 \\
\hline End BMI $\left(\mathbf{k g} / \mathbf{m}^{2}\right)$ & $45.4^{\star \star}$ & 5.6 & $46.7^{\star \star}$ & 6.3 & $44^{* *}$ & 6.5 \\
\hline $\begin{array}{l}\text { Change in BMI } \\
\left(\mathrm{kg} / \mathrm{m}^{2}\right)\end{array}$ & -1.9 & 1.9 & -2.2 & 1.7 & & 1.9 \\
\hline $\begin{array}{l}\text { Achieved } 5 \% \\
\text { weight loss }\end{array}$ & $47.9 \%$ & - & $53.2 \%$ & - & 33.3 & - \\
\hline $\begin{array}{l}\text { Achieved } 10 \% \\
\text { weight loss }\end{array}$ & $18.8 \%$ & - & $18.3 \%$ & & $8.3 \%$ & - \\
\hline $\begin{array}{l}\text { Achieved } 1 \text { BMI unit } \\
\text { loss }\end{array}$ & $64 \%$ & & & - & $58.3 \%$ & - \\
\hline $\begin{array}{l}\text { Completed } 50 \% \text { of } \\
\text { dietetic sessions }\end{array}$ & $95.8 \%$ & & $96.3 \%$ & - & $83.3 \%$ & - \\
\hline $\begin{array}{l}\text { Attended } 100 \% \text { of } \\
\text { dietetic sessions } \\
\text { including final F2F } \\
\text { session }\end{array}$ & & & $66.1 \%$ & - & $33.3 \%{ }^{*}$ & - \\
\hline $\begin{array}{l}\text { Psychology sessions } \\
\text { undertaken }\end{array}$ & 2.2 & 2.2 & 2.2 & 2 & $0.8^{*}$ & 1.7 \\
\hline $\begin{array}{l}\text { Prevalence of type } \\
2 \text { diabetes }\end{array}$ & $45.8 \%$ & - & $26.6 \%$ & - & $41.7 \%$ & - \\
\hline $\begin{array}{l}\text { Proportion using } \\
\text { orlistat }\end{array}$ & $2.1 \%$ & - & $5.5 \%$ & - & $0 \%$ & - \\
\hline $\begin{array}{l}\text { Proportion using } \\
\text { GLP-1 analogues }\end{array}$ & $8.3 \%$ & - & $6.4 \%$ & - & $16.7 \%$ & - \\
\hline $\begin{array}{l}\text { Proportion using } \\
\text { SGLT-2 inhibitors }\end{array}$ & $6.3 \%$ & - & $3.7 \%$ & - & $0 \%$ & - \\
\hline
\end{tabular}


227 Note: ${ }^{*}=$ Statistically significant compared to other interventions, assessed by RMANOVA (continuous 228 variables) or Pearson Chi-squared test (categorical variables): all $P<.001 ;{ }^{*}=$ Statistically significant compared to baseline, assessed by RMANOVA: all $P<.001$. Abbreviations: $B O C F=$ Baseline observation carried forward, BMI = Body Mass Index, GLP-1 = glucagon-like peptide-1, SGLT-2 = sodium-glucose co-transporter-2, SD = standard deviation.

\section{Core Programme Analysis}

233 RMANOVA revealed significant main effects of time for weight loss $\left(F_{1,166}=82.11, P<.001\right.$, 234 $\left.\eta^{2}=.33\right)$ and $\mathrm{BMI}\left(\mathrm{F}_{1,166}=87.39, P<.001, \eta^{2}=.34\right)$. However, there were no main effects for group and no interaction effects. As such, patients in all three treatment interventions significantly reduced body mass and BMI (all $P<.001$ ) compared to baseline, but there were no differences in the amount of change between interventions $(P=0.061)$. The mean weight loss for the Face-to-face intervention was $5.3 \pm 5.5 \mathrm{~kg}$ ( $4.1 \%$ of body mass), compared to 6.1 $\pm 4.9 \mathrm{~kg}(4.5 \%)$ for the App intervention, and $4 \pm 5.3 \mathrm{~kg}(3.4 \%)$ for the Phone intervention. Similarly, mean changes in BMI were $1.9 \pm 1.9,2.2 \pm 1.7$, and $1.5 \pm 1.9 \mathrm{BMI}$ units for the Faceto-face, App, and Phone interventions, respectively. Figure 2 shows the proportion of patients that achieved a reduction in body mass of $5 \%$ and $10 \%$, in addition to the proportion that had a reduction in body mass equivalent to one BMI unit or more. These data are summarised in table 1.

\section{2-week follow-up analysis}

246 Patient characteristics are summarised in table 2. RMANOVA revealed a significant main effect of time for weight loss $\left(F_{1.9,79.5}=25.65, P<.001, \eta^{2}=.48\right)$ with post-hoc analyses revealing that body masses were significantly lower at the end of the core programme and at the 12-week follow-up appointment (both $P<.001$ ) compared to baseline for both treatment interventions. However, there were no differences between the end of the core programme and 12-week follow up. Likewise, a significant main effect of time for $\mathrm{BMI}\left(\mathrm{F}_{1.8,78.7}=27.96, P\right.$ $\left.<.001, \eta^{2}=.29\right)$ revealed lower values at the end of the core programme and the 12 -week follow-up (both $P<.001$ ) compared to baseline for both interventions, with no differences between the end of the core programme and 12-week follow-up $(P=0.156)$, neither was there 
255 a statistically significant difference in weight losses between interventions from the start of the

256 core programme to the end of the 12 week follow up $(p=0.135)$. There were no significant main

257 effects for group or interaction effects. Figure $\mathbf{3}$ shows change in body mass for patients that

258 opted for and attended the 12-week follow-up.

259 Table 2 Patient characteristics at baseline, completion of the core programme and at 12 week follow 260 up of the programme for the Face-to-face and App interventions. Completer data is presented for both 261 the core programme and 12-week follow-up data.

\begin{tabular}{|c|c|c|c|c|c|c|}
\hline & \multicolumn{3}{|c|}{$\begin{array}{l}\text { Face-to-face } \\
\qquad(n=21)\end{array}$} & \multicolumn{2}{|r|}{$\begin{array}{c}\text { App } \\
(n=46)\end{array}$} & \multirow[b]{2}{*}{$\begin{array}{l}12 w \\
F U\end{array}$} \\
\hline & $\begin{array}{c}\text { Baseli } \\
\text { ne }\end{array}$ & $\begin{array}{l}\text { Core } \\
\text { Prog }\end{array}$ & $\begin{array}{l}12 w \\
F U\end{array}$ & $\begin{array}{c}\text { Baseli } \\
\text { ne }\end{array}$ & $\begin{array}{l}\text { Core } \\
\text { Prog }\end{array}$ & \\
\hline Stature $(m)$ & $\begin{array}{c}1.66 \pm \\
0.08\end{array}$ & & & $\begin{array}{c}1.67 \pm \\
0.09\end{array}$ & - & - \\
\hline Age (years) & $\begin{array}{r}52.9 \\
3.1\end{array}$ & & - & $\begin{array}{c}40.8 \pm \\
11.6\end{array}$ & - & - \\
\hline Mass (kg) & 17 & $\begin{array}{c}122.6 \pm \\
15.8^{\star}\end{array}$ & $\begin{array}{c}122.3 \pm \\
16.7^{\star}\end{array}$ & $\begin{array}{c}138.3 \pm \\
22.6\end{array}$ & $\begin{array}{c}130.2 \pm \\
22.6^{*}\end{array}$ & $\begin{array}{c}129.1 \pm \\
23.4^{*}\end{array}$ \\
\hline $\begin{array}{l}\text { Change in mass } \\
\text { compared to } \\
\text { baseline }(\mathrm{kg})\end{array}$ & - & $\begin{array}{c}-7.3 \pm \\
5.6\end{array}$ & $\begin{array}{c}-7.6 \pm \\
9.3\end{array}$ & - & $\begin{array}{c}-7.9 \pm \\
4.8\end{array}$ & $\begin{array}{c}-9.2 \pm \\
7.6\end{array}$ \\
\hline $\begin{array}{l}\text { Percent change in } \\
\text { mass compared to } \\
\text { baseline }\end{array}$ & - & 5.5 & 5.6 & - & 5.9 & 6.8 \\
\hline BMI $\left(\mathbf{k g} / \mathrm{m}^{2}\right)$ & $\begin{array}{c}47.4 \pm \\
6.2\end{array}$ & $\begin{array}{c}44.7 \pm \\
5.5\end{array}$ & $\begin{array}{c}44.6 \pm \\
5.4\end{array}$ & $\begin{array}{c}49.4 \pm \\
6.9\end{array}$ & $\begin{array}{c}46.5 \pm \\
7\end{array}$ & $\begin{array}{c}46.1 \pm \\
7.2\end{array}$ \\
\hline $\begin{array}{l}\text { Change BMI units } \\
\text { compared to } \\
\text { baseline }\left(\mathrm{kg} / \mathrm{m}^{2}\right)\end{array}$ & - & $-2.7 \pm 2$ & $\begin{array}{c}-2.8 \pm \\
3.3\end{array}$ & - & $\begin{array}{c}-2.9 \pm \\
1.6\end{array}$ & $\begin{array}{c}-3.3 \pm \\
2.8\end{array}$ \\
\hline
\end{tabular}


Achieved 5\%

weight loss

Achieved 10\%

weight loss

Achieved 1 BMI

unit loss
66.7

47.6

71.7

60.9

28.6

23.8

26.1

23.9

85.7

76.2

89.1

87
262

Note: ${ }^{*}=$ Statistically significant compared to baseline, assessed by RMANOVA: all $P<.001$. Data are presented as mean \pm standard deviation unless indicated otherwise. Abbreviations: $\mathrm{BOCF}=$ Baseline observation carried forward, $\mathrm{BMI}=$ Body Mass Index.

\section{Intervention engagement and service satisfaction}

The number of psychology support sessions undertaken by the patients in the Phone group was significantly less than for the other groups $(0.8 \pm 1.7$ vs $2.2 \pm 2.2$ for the Face-to-face, and $2.2 \pm 2$ for the App group) $(P=.03)$, but the association between weight loss and number of psychology support sessions $(r=0.58)$ did not reach statistical significance. Intervention adherence, in terms of the number of patients completing half of the dietetic sessions was 95.8\%, 96.3\% and $83.3 \%$ for the Face-to-face, App and Phone interventions, respectively; $85.4 \%, 66.1 \%$ and $33.3 \%$ of patients attended all the dietetic sessions. Service satisfaction, as measured by the family and friends test score out of 10 , was administered by text message. There were no significant differences in responses between Face-to-face (10 \pm 0$)$, App (9.6 \pm $0.8)$ and Phone $(10 \pm 0)(P=.261)$; forty-two participants responded to the text message, giving a response rate of $24.9 \%$.

\section{DISCUSSION}

The premise for advocating digital healthcare as part of weight management support for people who are affected by overweight or obesity is predicated on the assumption that it can 
improve efficiency, accessibility, and personalisation of healthcare services. Fundamentally,

283 whether effectiveness in real-world settings is comparable or superior to more traditional 284 interventions, such as face-to-face delivery, could be decisive in terms of its value as a public health strategy. To the authors' knowledge, this is the first evaluation of part of a locally commissioned tier 3 weight management programme offering a range of interventions including digital support in everyday practice. The main findings were that Face-to-face, App and Phone interventions all resulted in significant weight loss compared to baseline values. Furthermore, differences between the interventions did not reach statistical significance meaning that a similar amount of weight loss was achieved using these approaches, including digital healthcare. Pragmatically, this could increase the weight management treatment options by reducing inconvenience, time burden and travel for individuals who otherwise might not engage with traditional face-to-face support, and from a healthcare delivery perspective offers cost-savings on resources such as clinic rooms and clinician travel time including instances where patients did not attend appointments.

Modest weight loss ( $~ 5 \%$ of initial body mass) induces clinically significant health benefits in risk factors for cardiovascular disease and type 2 diabetes. ${ }^{21-22}$ In the present service evaluation, half of all patients (i.e. from all three interventions) achieved this criterion. More patients in the App intervention (53\%) achieved the 5\% weight loss target compared to the other interventions (48\% for Face-to-face and 33\% for Phone, respectively) although the difference between groups did not reach statistical significance. Similarly, modest decreases in BMI might have an important impact on population health. It has been suggested that weight reduction to the magnitude of approximately one BMI unit, can reduce the risk of all-cause mortality by $6 \%$, and provide significant risk reduction as both a preventative and therapeutic treatment for type 2 diabetes. ${ }^{19}$ In this study, the number of patients in the App intervention that reduced their initial mass by one BMI unit or more was $74 \%$, compared to $64 \%$ for the Face-to-face group, and $58 \%$ for the Phone group. These data compare favourably to other weight management programmes commissioned by the $\mathrm{NHS}^{23-25}$ and to self-referring, fee- 
paying commercial weight management programmes. ${ }^{26}$ Yet, this also highlights the challenges

310 facing weight management services because around one in two patients did not reach this

311 weight loss target, which is consonant with meta-analyses for commercial weight loss

312 programmes. ${ }^{27}$ The use of Total Diet Replacements have demonstrated significant weight

313 losses in the context of weight management and diabetes remission services ${ }^{28-29}$; it is therefore

314 likely that their provision within this programme would have increased weight losses achieved,

315 highlighting the key role that this dietary intervention can play in driving further and more

316 significant weight losses within weight management services.

317 Nevertheless, the importance of modest weight loss should not be underestimated. The

318 prevalence of diabetes is known to increase in a linear fashion as BMI levels increase with a 319 prevalence rate of up to one quarter for populations with severe obesity (i.e. $\mathrm{BMI} \geq 40 \mathrm{~kg} / \mathrm{m}^{2}$ ). ${ }^{30}$

320 Furthermore, data collected for the Health Survey for England demonstrated that half of the 321 population who have a $\mathrm{BMI}>25 \mathrm{~kg} / \mathrm{m}^{2}$ and $\geq 40$ years of age have prediabetes. ${ }^{31}$ Therefore,

322 it is encouraging that the strongest associations between changes in weight loss and health

323 outcomes are frequently seen for glycaemic control, with clinically significant improvements 324 observed with as little as $2 \%$ to $<5 \%$ reduction in initial mass. ${ }^{32}$ Although weight losses of 325 greater magnitude are associated with greater improvements in risk factors, ${ }^{32}$ it is likely that more than half of patients who opted to receive the App intervention in the present service evaluation benefitted from clinically significant changes in important health outcomes. Given that the interventions in the current programme were relatively short, it is possible that greater weight losses would have been achieved with a longer intervention, as per the findings of other studies $^{33}$, however due to the short-follow up period in this study we were unable to validate longer-term outcomes. Forty-one percent of patients chose to attend the 12-week follow-up appointment. Patients were able to maintain weight loss, but there were no further significant reductions in body mass at this point.

334 The average baseline BMI $\left(48.3 \mathrm{~kg} / \mathrm{m}^{2}\right)$ in this study was higher than those reported in 335 previous studies, ${ }^{25-27}$ and is in line with guidelines for referral to tier 3 weight management 
services. A novel finding therefore is that dietetic interventions using traditional delivery

337 mechanisms such as face-to-face support or smartphone apps can also be effective for

338 individuals who are affected by severe obesity, at least over a period of 4-5 months.

339 Considering that digital healthcare has possible advantages compared to other modes of

340 support, such as greater time- and cost-effectiveness and potential for broad dissemination

341 and scalability, ${ }^{34}$ it could improve the capacity of NHS-led programmes to support people that

342 are obese. In the current service, almost two thirds of participants opted for the App

343 intervention revealing the broad appeal of this method of support, which is conceivably

344 unsurprising considering that $79 \%$ of UK adults personally use a smartphone. ${ }^{35}$ The mean age

345 of patients that self-selected the App intervention was significantly lower than for the other

346 treatment groups, which supports evidence that younger demographics are the main users of

347 health apps. ${ }^{36}$ Uptake of the weight management programme was higher for women than men as seen elsewhere ${ }^{24}$, and a similar proportion of men and women chose each intervention.

349 Rigorous clinical interventions for obesity typically require face-to-face contact but frequently suffer from low adherence and high attrition, ${ }^{12,19}$ although some evidence suggests that smartphone apps can increase engagement and adherence particularly through the provision of frequent tailored feedback. ${ }^{37}$ Nonetheless, in the current intervention, adherence (those attending $100 \%$ of dietetic sessions) was greater for the Face-to-face group compared to the App group, although there were no significant differences in attrition rates (number of patients lost to follow up) (4.2\% and 6.4\% for the Face-to-face and App groups, respectively). Despite this, there was no difference seen in weight loss outcomes. The final session for all pathways was a face-to-face session, so if patients completed the app or telephone sessions but did not attend the final face-to-face session, they were not considered to have attended $100 \%$ of the sessions. This may offer reason as to why numbers regarding $100 \%$ attendance were lower in the App and Phone group if they did not want to or it was not convenient to attend face-toface, which suggests potential usefulness in a fully remote pathway to encourage attendance of all sessions within a programme. The Phone group had significantly lower adherence and 
greater attrition rates (42\%) compared to the other treatment options, suggesting this type of

364 intervention may be less effective in real-world settings, since adherence is considered a

365 prerequisite for the success of behavioural interventions. However, due to the small sample in the Phone intervention it is difficult to draw conclusions regarding the clinical effectiveness for this group. Patient satisfaction for the service was rated highly for the App group (mean score of $9.6 \pm 0.8$ out of 10 ) and was no different to the Face-to-face group, although the response to the FFT was voluntary and was not received from all individuals. Taken together, these data demonstrate that the impact and acceptability of the weight management service compare well against long-term obesity and overweight pharmacotherapy, ${ }^{38-39}$ and that smartphone app support is at least as effective as traditional behaviour change techniques. ${ }^{9}$ We have shown that digital support can produce quantitatively significant impact on weight outcomes comparable to more resource-intensive, face-to-face approaches in free-living patients.

375 There are several limitations which should be considered alongside the findings of this study.

376 As this was a service evaluation, a control group was not included, and results were based on 377 people who were 'ready to change' by opting to join a weight management service. As such, they cannot be considered a random sample of the population affected by obesity. 379 Furthermore, the interventions were only carried out in one geographical region, West Yorkshire, and might not be nationally representative. Patients were also able to choose their treatment intervention according to preference which resulted in unequal sample sizes for the three groups. This can reduce statistical power and increases the likelihood of a type I error. ${ }^{40}$

383 The proportion of patients with type 2 diabetes was lower in the App intervention, although 384 this likely reflects the lower mean age for this group. A further limitation is that patients were not followed up at 12 months, because this was beyond the remit of the current evaluation. Furthermore, it should be noted that the 12-week follow-up data is not representative of the full cohort, just those who requested the additional follow-up or those who wanted to pursue bariatric surgery. Nevertheless, this paper provides valuable preliminary data and we 
recommend that future research considers the longer-term effects on weight loss maintenance when comparing different modes of service delivery including digital care provision.

391 There are also several strengths of the current study that should be discussed. The purpose was not to compare the efficacy of the weight management service with other treatments, but rather to consider its effectiveness as it runs in practice with people aiming to reduce their body mass in everyday life. This is important because interventions to improve health frequently bring about intended effects under highly controlled circumstances but often fail to demonstrate benefits in real-world contexts, and this dissonance is frequently overlooked. Furthermore, weight outcomes for digital healthcare interventions are often likely to be assessed via self-report and should be interpreted with caution, but were recorded by healthcare professionals in the current service evaluation at the end of the core programme and the optional 12-week follow-up. The results are also based on a locally commissioned tier 3 weight management service using a population of patients with severe obesity with a higher average BMI compared to previously published evaluations. Additionally, we have presented data using BOCF for the core programme analysis, as opposed to complete case data. Although all imputation methods have limitations, BOCF provides a more conservative approach for assessing service effectiveness and is recommended for quantifying weight loss outcomes. $^{9,41}$

In conclusion, by undertaking the Way to Wellness weight management programme, around one half of patients achieved clinically meaningful weight loss of $5 \%$ from baseline. The patient interventions and delivery modes were chosen by patients in a real-life setting showing that one size may not fit all, so having intervention options may be advantageous. There were no differences between treatment groups meaning that on average patients lost similar amounts of weight regardless of which intervention they used. Alternative dietary interventions, such as

413 total diet replacements could also be considered in such services to drive further weight

414 losses. The smartphone application intervention was well received with acceptable adherence 415 and attrition rates compared to more traditional face-to-face support. As such, we provide 
416 evidence to suggest that digital healthcare deserves a prominent place among a variety of

417 dietetic support options that can be used to bring about weight loss in everyday practice. This

418 might prove valuable if such interventions can circumvent some of the resource and additional

419 costs attributed to face-to-face support. Digital care provision offers potential to scale which

420 could yield great benefits, even when more modest weight losses are achieved. Future

421 research should aim to establish the long-term effects including clinical outcomes such as

422 blood pressure, lipids, and blood glucose levels.

$423 \quad$ Conflicts of interest statement

424 R.H and L.J are employees of Oviva UK Ltd. Data analysis was completed independently by

425 M.H at the University of Huddersfield who declares no conflict of interest.

\section{$426 \quad$ ICMJE-generated Conflict of interest statements}

427 Dr. Huntriss reports other from Oviva, during the conduct of the study; .

428 Dr. Haines has nothing to disclose.

429

Dr. Jones reports other from Oviva UK Ltd, during the conduct of the study; .

430 Acknowledgements (including author contributions)

431 R. H. was responsible for data collection. M.H. was responsible for data analyses. R.H. and 432 M.H. were responsible for data interpretation and writing the manuscript. L.J. reviewed the 433 data collection, analysis and manuscript.

434 We would like to thank the clinical and administrative teams at Oviva UK Ltd and Wakefield

435 Council for their inputs surrounding service delivery. 
1. NHS Digital. Statistics on Obesity, Physical Activity and Diet, England, 2019. Accessed 29 April 2020. https://digital.nhs.uk/data-andinformation/publications/statistical/statistics-on-obesity-physical-activity-anddiet/statistics-on-obesity-physical-activity-and-diet-england-2019/part-3-adult-obesity

2. Branca F, Nikogosian H, Lobstein T. World Health Organization: The challenge of obesity in the WHO European Region and the strategies for response. Accessed 29 April 2020. http://www.euro.who.int/ data/assets/pdf file/0008/98243/E89858.pdf

3. Public Health England. Health matters: obesity and the food environment. Accessed 29 April 2020. https://www.gov.uk/government/publications/health-matters-obesityand-the-food-environment/health-matters-obesity-and-the-food-environment--2

4. British Obesity and Metabolic Surgery Society. Commissioning guide: weight assessment and management clinics (tier 3). Accessed 29 April 2020. https://www.bomss.org.uk/wp-content/uploads/2017/10/Revision-of-Commissioningguide-Tier-3-clinics-04042017.pdf

5. NICE. Obesity: identification, assessment and management. Clinical guideline [CG189]. Accessed 29 April 2020. https://www.nice.org.uk/guidance/cg189/chapter/1-Recommendations\#ftn.footnote 6

6. Fountain D, Alkharaiji M, Awad S, Hughes D, Idris I. Prevalence of co-morbidities in a specialist weight management programme prior to bariatric surgery. $\mathrm{Br} J$ Diabetes. 2019;19:8-13.

7. Kininmonth AR, Bradbury J. Evaluation of a Tier 3 Specialist Weight Management Service for morbidly obese patients. Proc Nutr Soc. 2016; 75; E203.

8. Jennings A, Hughes CA, Kumaravel B, et al. Evaluation of a multidisciplinary Tier 3 weight management service for adults with morbid obesity, or obesity and comorbidities, based in primary care. Clin Obes. 2014; 4(5): 254-266.

9. Hartmann-Boyce J, Johns DJ, Jebb SA. Effect of behavioural techniques and delivery mode on effectiveness of weight management: systematic review, metaanalysis and meta-regression. Obes Rev. 2014;15(7):598-609.

10. Gilmore LA, Duhé AF, Frost EA, Redman LM. The technology boom: a new era in obesity management. J Diabetes Sci Technol. 2014; 8: 596-608.

11. NICE. Behaviour Change: individual approaches. Public health guideline [PH49]. Accessed 1 November 2020]. https://www.nice.org.uk/guidance/ph49/resources/behaviour-change-individualapproaches-pdf-1996366337989

12. Rivera J, McPherson A, Hamilton J, et al. Mobile Apps for Weight Management: A Scoping Review. JMIR Mhealth Uhealth. 2016; 4(3): e87. 
13. The NHS Long Term Plan. Accessed 29 April 2020. https://www.longtermplan.nhs.uk/wp-content/uploads/2019/08/nhs-long-term-planversion-1.2.pdf

14. Liu F, Kong X, Cao S, et al. Mobile phone intervention and weight loss among overweight and obese adults: a meta-analysis of randomized controlled trials. Am J Epidemiol. 2015;181(5): 337-48

15. Schippers M, Ada PC, Smolenski DJ, Wong HT, de Wit JB. A meta-analysis of overall effects of weight loss interventions delivered via mobile phones and effect size differences according to delivery mode, personal contact, and intervention intensity and duration. Obes Rev. 2017;18(4):450-459.

16. Park S-H, Hwang J, Choi Y-K. Effect of Mobile Health on Obese Adults: A Systematic Review and Meta-Analysis. Healthc Inform Res. 2019; 25(1): 12-26.

17. Peek N, Sujan M, Scott $P$. Digital health and care in pandemic times: impact of COVID-19. BMJ Health Care Inform. 2020; 27:e100166. doi: 10.1136/bmjhci-2020100166

18. Murray E, Hekler EB, Andersson G, et al. Evaluating digital health interventions: key questions and approaches. Am J Prev Med. 2016; 51(5): 843-851.

19. Hartmann-Boyce J, Johns DJ, Jebb SA. Behavioural weight management programmes for adults assessed by trials conducted in everyday contexts: systematic review and meta-analysis. Obes Rev. 2014;15(11):920-32.

20. NICE. Weight management: lifestyle services for overweight or obese adults. Public health guideline [PH53]. Accessed 04 May 2020. https://www.nice.org.uk/guidance/ph53

21. Klein S, Burke LE, Bray GA, et al. Clinical implications of obesity with specific focus on cardiovascular disease. Circulation. 2004;110(18):2952-2967.

22. Lloyd A, Khan R. Evaluation of Healthy Choices: a commercial weight loss programme commissioned by the NHS. Perspect Public Health. 2011;131(4):177-83.

23. Van Gaal LF, Mertens IL, Ballaux D. What is the relationship between risk factor reduction and degree of weight loss? Eur Heart J Suppl. 2005;7:L21-L26.

24. Ahern AL, Olson AD, Aston LM, Jebb SA. Weight Watchers on prescription: An observational study of weight change among adults referred to Weight Watchers by the NHS. BMC Public Health. 2011;11:434.

25. Jolly $\mathrm{K}$, Lewis A, Beach J, et al. Comparison of range of commercial or primary care led weight reduction programmes with minimal intervention control for weight loss in obesity: Lighten Up randomised controlled trial. BMJ. 2011;343: doi: 10.1136/bmj.d6500 
26. Stubbs RJ, Morris L, Pallister C, Horgan G, Lavin JH. Weight outcomes audit in 1.3 million adults during their first 3 months' attendance in a commercial weight management programme. BMC Public Health. 2015;15:882.

27. McEvedy SM, Sullivan-Mort G, McLean SA, et al. Ineffectiveness of commercial weight-loss programs for achieving modest but meaningful weight loss: Systematic review and meta-analysis. J Health Psychol. 2017;22(12):1614-1627.

28. Lean ME, Leslie WS, Barnes AC, et al. Primary care-led weight management for remission of type 2 diabetes (DiRECT): an open-label, cluster-randomised trial. Lancet. 2018; 391(10120):541-551.

29. Astbury NM, Aveyard P, Nickless A, et al. Doctor Referral of Overweight People to Low Energy total diet replacement Treatment (DROPLET): pragmatic randomised controlled trial. BMJ. 2018; 362:k3760.

30. Bays HE, Chapman HR, Grandy S. The relationship of body mass index to diabetes mellitus, hypertension and dyslipidaemia: comparison of data from two national surveys. Int J Clin Pract. 2007;61(5): 737-747.

31. Mainous III AG, Tanner RJ, Baker R, et al. Prevalence of prediabetes in England from 2003 to 2011: population-based, cross-sectional study. BMJ Open. 2014;4(6):e005002.

32. Wing R, Lang W, Wadden TA, et al. Benefits of modest weight loss in improving cardiovascular risk factors in overweight and obese individuals with type 2 diabetes. Diabetes Care. 2011;34:1481-1486.

33. Laws R. A new evidence-based model for weight management in primary care; the Counterweight Programme. J Hum Nutr Diet. 2004;17(3):191-208.

34. Carter MC, Burley VJ, Nykjaer C, Cade JE. Adherence to a smartphone application for weight loss compared to website and paper diary: pilot randomized controlled trial. J Med Internet Res. 2013;15(4):e32.

35. OfCom. Communications Market Report 2019. Accessed 13 May 2020. https://www.ofcom.org.uk/ data/assets/pdf file/0020/117065/communicationsmarket-report-2019.pdf

36. Carroll JK, Moorhead A, Bond R. Who Uses Mobile Phone Health Apps and Does Use Matter? A Secondary Data Analytics Approach. J Med Internet Res. 2017;19(4):e125.

37. Dounavi K, Tsoumani O. Mobile Health Applications in Weight Management: A Systematic Literature Review. American Journal of Preventive Medicine. 2019;56(6):894-903.

38. Yanovski SZ, Yanovski JA. Long-term Drug Treatment for Obesity: A Systematic and Clinical Review. JAMA. 2014;311(1):74-86. 
557

558

559

560

561

562

563

564

565

566

567

568

569

570

571

572

573

574

575

576

577

578

579

580

581

582

583

584

585

586

587

588

39. Torgerson JS, Hauptman J, Boldrin MN, Sjöström L. XENical in the prevention of diabetes in obese subjects (XENDOS) study: a randomized study of orlistat as an adjunct to lifestyle changes for the prevention of type 2 diabetes in obese patients. Diabetes Care. 2004;27(1):155-61.

40. Rusticus S, Lovato C. Impact of Sample Size and variability on the Power and Type I Error Rates of Equivalence Tests: A Simulation Study. Practical Assessment, Research \& Evaluation. 2014;19: doi 10.7275/4s9m-4e81

41. Shao J, Jordan DC, Pritchett YL. Baseline observation carry forward: reasoning, properties, and practical issues. J Biopharm Stat. 2009;1

(1) 
Fig 1. CONSORT style diagram to illustrate allocation and intervention phases. BOCF used for core programme analysis. Completer data used for 12-week follow-up analysis.

609

610

611

612

613

614

615

616

617

618
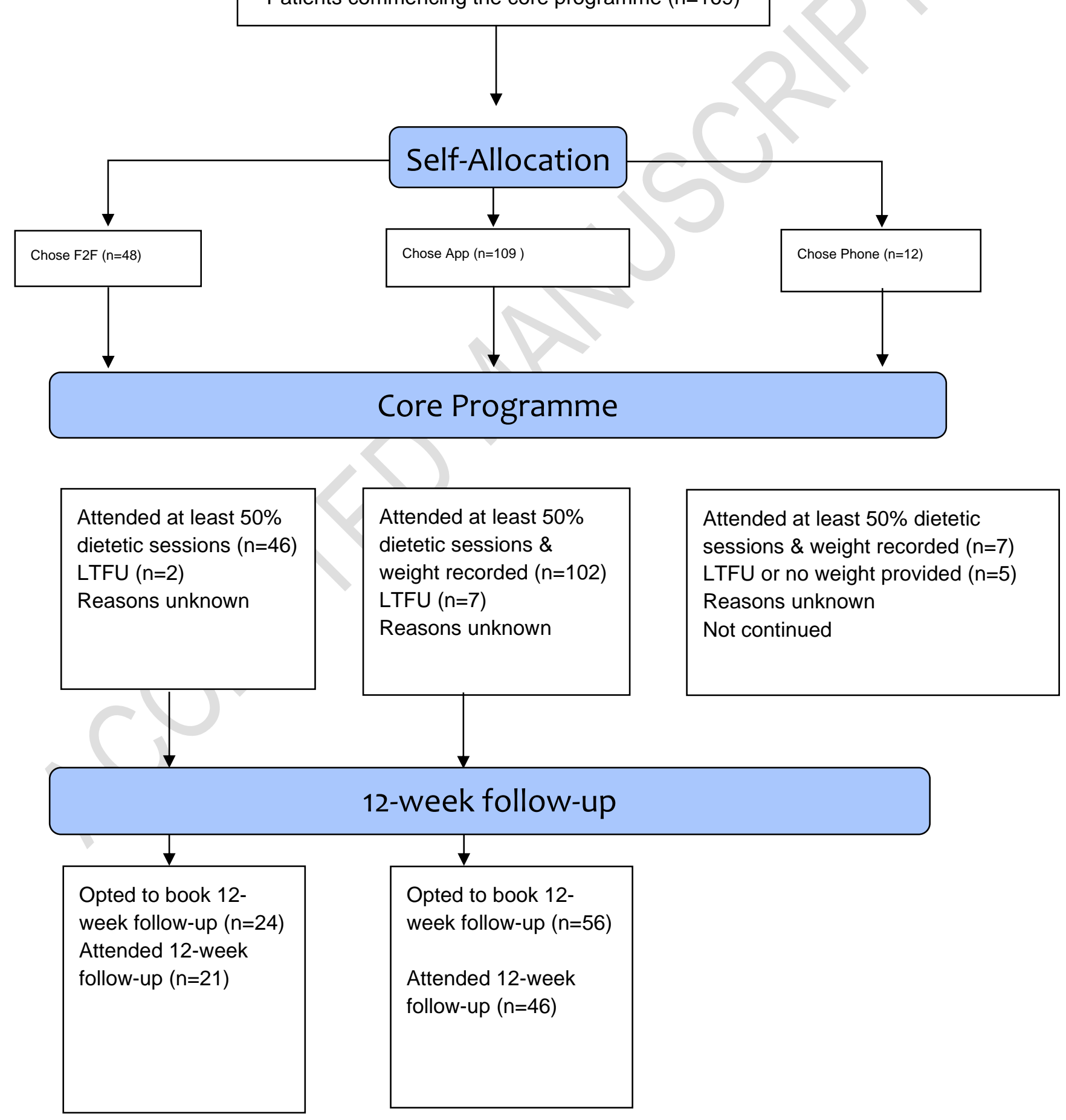


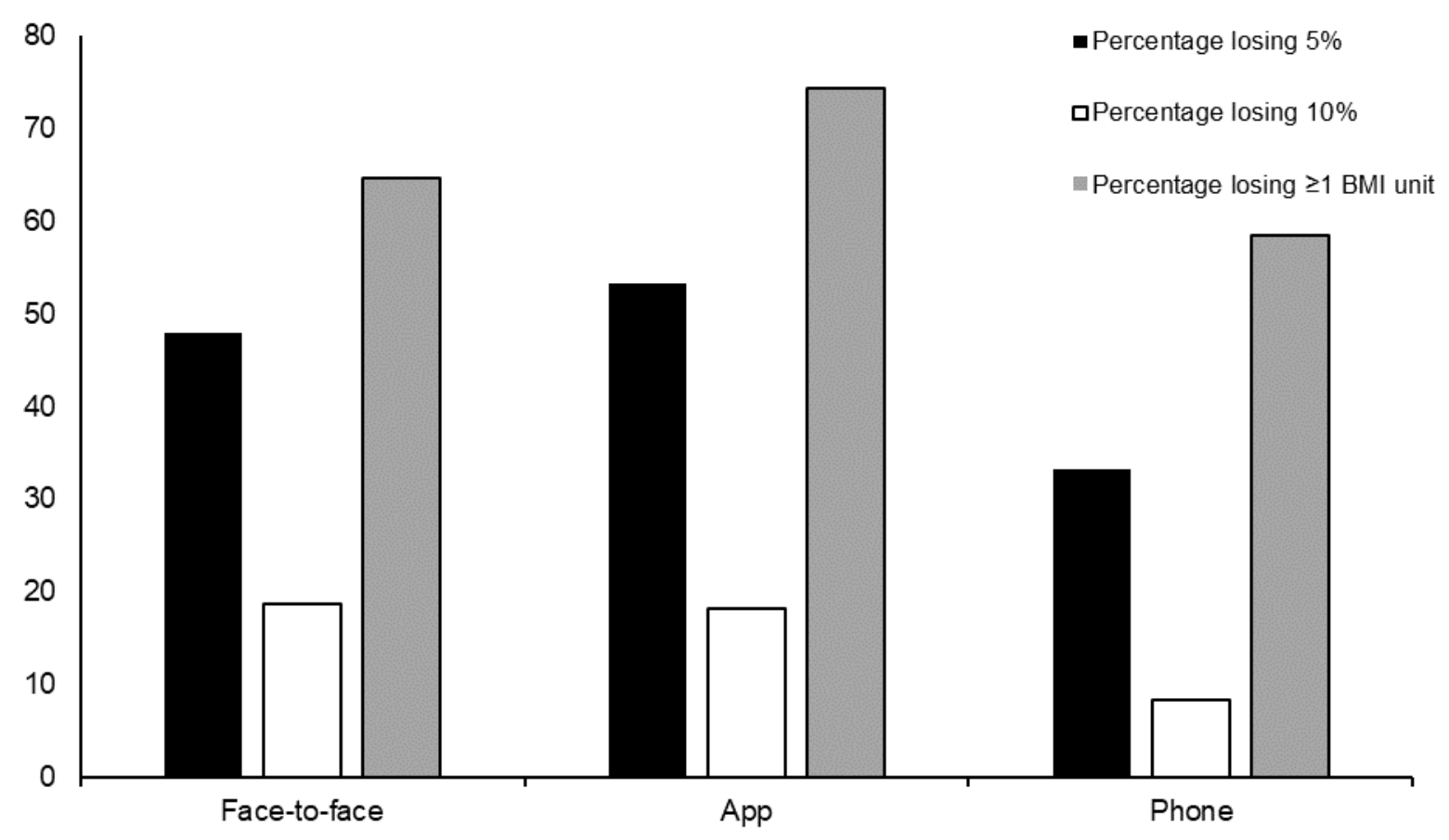

636

637

638

639

640

641

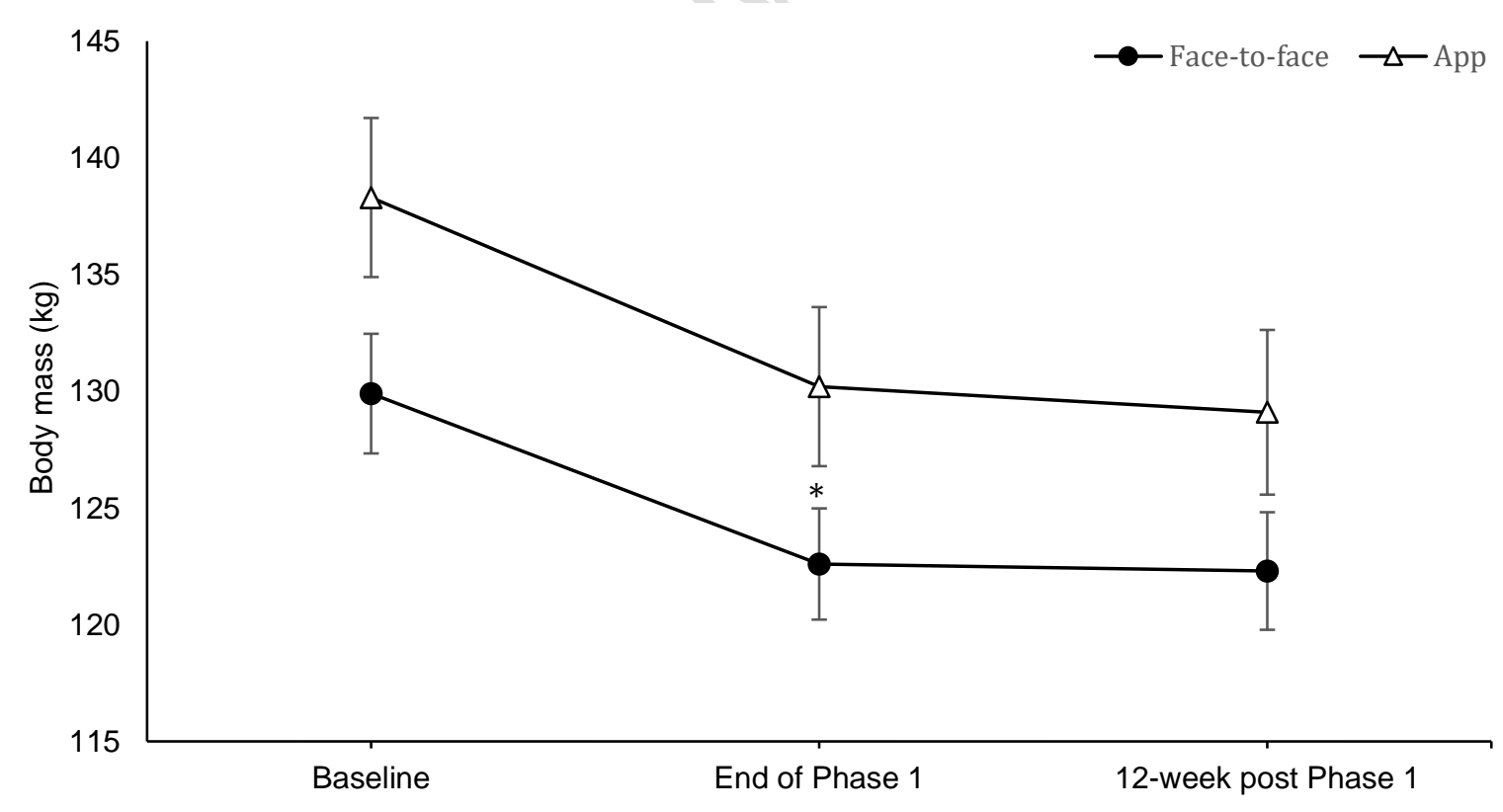

642 